$\sum$ the same firm foundation on the best available science and potentially an even stronger mandate for action.

From the outset, the IPAMR needs to avoid simply restating the problem. It must move rapidly to an agenda that includes identifying key knowledge gaps and how

商 to fill them; assessing viable short- and

Oo long-term solutions; evaluating barriers to implementation; and setting out road maps for sustainable control of diseasecausing microbes. It could, for example, support studies to investigate dosing regimes that stall resistance, coordinate incentives for developing new types of antimicrobial and set targets for prescriptions and animal use.

To have any chance of achieving these objectives, the IPAMR must be trusted and free of vested interests. It will need to involve a broad range of experts, encompassing clinical and veterinary medicine, epidemiology, microbiology, pharmacology, health economics, international law and social science. It will need technical, financial, industrial and political support from governments and agencies including the WHO, the World Organisation for Animal Health, the World Trade Organization and the United Nations, as well as from representatives of producers and consumers of antimicrobial drugs. Above all, it will need strong, independent leadership.

Creating an effective IPAMR will be a huge undertaking, but the successful global campaign to eradicate smallpox, led by the WHO, demonstrates that a coordinated, international response to a public-health threat can work. The attempt must be made - otherwise, the massive health gains made possible by antimicrobial drugs will be lost.

Mark Woolhouse is professor of infectious disease epidemiology in the Centre for Immunity, Infection \& Evolution at the University of Edinburgh, $U K$. Jeremy Farrar is director of the Wellcome Trust, London, UK.

e-mails:mark.woolhouse@ed.ac.uk; j.farrar@wellcome.ac.uk

1. World Health Organization Antimicrobial Resistance: Global Report on Surveillance 2014 (WHO, 2014).

2. Harris, S. R et al Science 327, 469-474 (2010).

3. Vernet, G. et al. Emerg. Inf. Dis. 20, 434-440 (2014).

4. Laxminarayan, R. et al. Lancet Inf. Dis. 13, 1057-1098 (2013)

5. Wertheim, H. F. L. et al. PLoS Med. 10, e1001429 (2013).

6. D'Costa, V. M. et al. Nature $4 \mathbf{7 7}, 457-461$ (2011).

7. Whitman, W. B. et al. Proc. Natl Acad. Sci. USA 95, 6578-6583 (1998)

8. Cooper, M. A. \& Shlaes, D. Nature 472, 32 (2011).

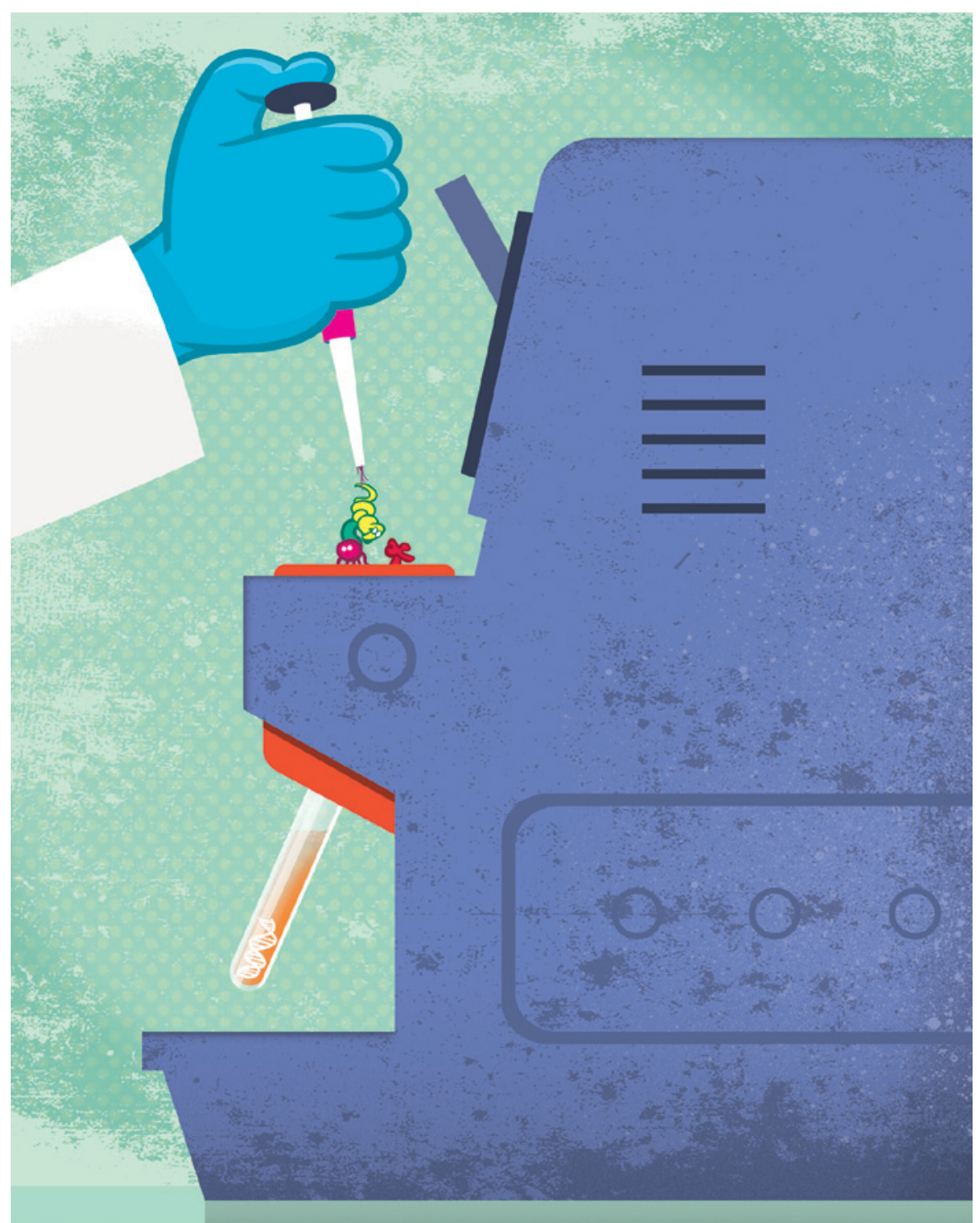

\title{
Bring microbial sequencing to hospitals
}

\section{Analysing bacterial and viral DNA can help doctors to pick effective drugs quickly, says Sharon Peacock.}

A patient goes to her doctor with fever, cough and night sweats. Rapid tests confirm the diagnosis of tuberculosis and hint at multidrug resistance. But to suggest the optimum drug combination, as many as eight weeks of laboratory testing are required - a timescale dictated by the slow growth rate of the pathogen (Mycobacterium tuberculosis). In the meantime, the doctor must make an educated guess about which medicines to prescribe, increasing the risk of ineffective treatment and spread of infection.

Yet it would take less than a week to 
sequence a culture of $M$. tuberculosis and to detect mutations that indicate which drugs the bacteria are resistant to. My colleagues and I demonstrated ${ }^{1}$ this in a research setting last year using a sputum culture from a tuberculosis patient. We have also shown that whole-genome sequencing can detect resistance of other pathogens to carbapenem antibiotics, drugs reserved to treat the most serious infections. Although a range of genetic mechanisms can confer resistance, sequencing is informative in all species tested so far, including Acinetobacter baumannii and Klebsiella pneumoniae, which can infect the most vulnerable hospital patients ${ }^{2}$. Sequence information can also be used to confirm outbreaks and help to bring them to a close.

Although technology for microbial sequencing has existed for years, it is yet to help patients on a routine basis. Now that pathogen genomes can be completed in less than a day, the time is right to begin using them to control and treat serious infections, at least in the developed world. This will take two developments: the introduction of sequencing into local diagnostic laboratories and the creation of automated tools to interpret newly sequenced genomes. Both are more a matter of will than of innovation.

\section{USEFUL DATA}

A baby born in 1930 in the United States had a life expectancy of around 60 years; someone born today has a life expectancy of about 80 . By some estimates, antibiotics have contributed as much as a decade to that jump in lifespans. Now the

rise of antimicrobial resistance threatens these gains ${ }^{3}$. In a post-antibiotic era, even minor infections could prove fatal. Worse, without effective antibiotics, most medical practices, including routine surgery, emergency operations, transplants and chemotherapy, will be less safe.

Microbial sequencing could help physicians to know which antibiotics will be effective for their patients. Although it

"In a postously undocumented antibiotic era, forms of resistance, evenminor a genome sequence infections can simultaneously could prove reveal mutations and fatal." acquired genes that bestow resistance to many antibiotics. By contrast, real-time polymerase chain reaction (PCR), a more limited form of DNA analysis, can detect no more than a handful of known resistance markers in a sample. Our 2013 sequencing analysis ${ }^{1}$ showed that a patient with tuberculosis was infected with a mixture of two strains, both resistant to more than a dozen antibiotics including some that are not routinely evaluated (see 'Resistance on the rise').

Several research groups have used sequencing to trace outbreaks of multidrug resistant pathogens in hospitals. For example, a retrospective genomic investigation $^{4}$ of a 2011 outbreak of carbapenemresistant K. pneumoniae at the US National Institutes of Health Clinical Center in Bethesda, Maryland, helped researchers to reconstruct the most likely transmission routes and revealed a network that included

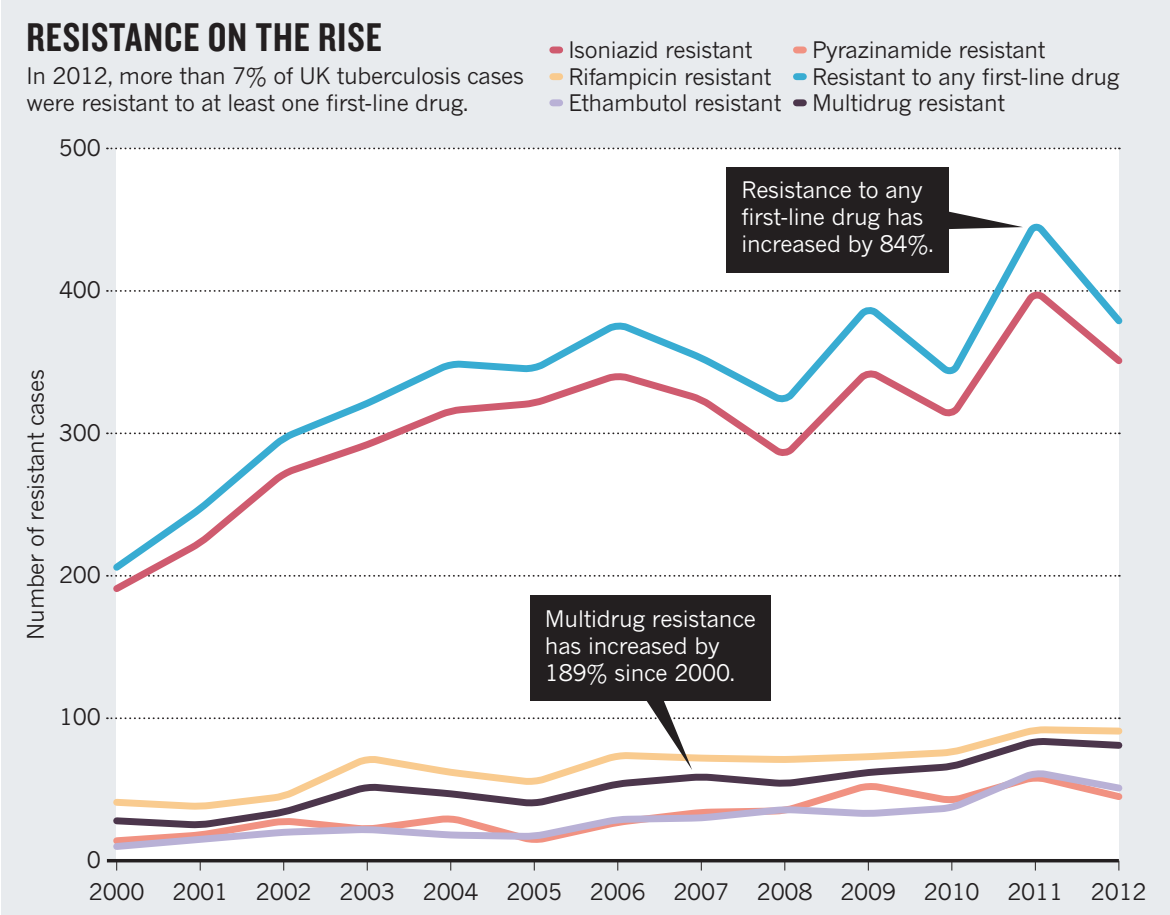

asymptomatic patients and contaminated medical equipment. This could guide targeted interventions including cleaning and sterilizing procedures.

\section{NEED FOR SPEED}

Human genomes capture the limelight in most discussions about the benefits of and barriers to bringing sequencing into health care. Yet pathogen sequencing represents a quick win. Bench-top sequencers that cost around US\$125,000 can complete several bacterial genomes in a day, at a cost of around $\$ 150$ per sample - about twice as much as running some commercial PCR tests that detect resistance to one drug at a time.

People working to bring human sequencing to the clinic generally aim to establish large, centralized facilities to control costs, maintain quality and facilitate data sharing. A 15-day turnaround or better is the target by the end of the 5-year Genomics England programme, which aims to sequence 100,000 human genomes by 2017 , according to Mark Caulfield, the programme's chief scientist. This timescale is tolerable because the data rarely relate to conditions that are immediately life-threatening.

With infectious diseases, however, swift answers really matter. Suspected outbreaks can be confirmed and nipped in the bud. Within an hour of receiving results, clinicians could tailor treatments of hospitalized patients, providing the right drug at the right dose and route of administration. This precision will help more people than just the patient. Infections that are cured quickly are less likely to spread. It also means using fewer ineffective drugs, reducing the selective pressure for resistance, and leading to fewer pointlessly disrupted gut microbiomes.

Therefore, microbial sequencing should be done as close to the patient as possible, avoiding delays from packaging and shipping. This is feasible. Clinical microbiology laboratories are in place across the developed world, and have a track record of embracing new technologies. An example is the uptake of mass spectrometry starting five or so years ago to identify pathogens based on tell-tale microbial peptides. Diagnostic laboratories have also adopted real-time PCR, which entered clinical diagnostic laboratories about a decade ago.

Existing mechanisms to develop standard operating procedures and accreditation are applicable to microbial sequencing. Diagnostic laboratories already have tightly controlled systems to collect, track and process samples. They have defined turnaround times, they can link test results to patient and infection-control information, and they use an information system that ensures patient confidentiality.

Sequencing for HIV is already established 


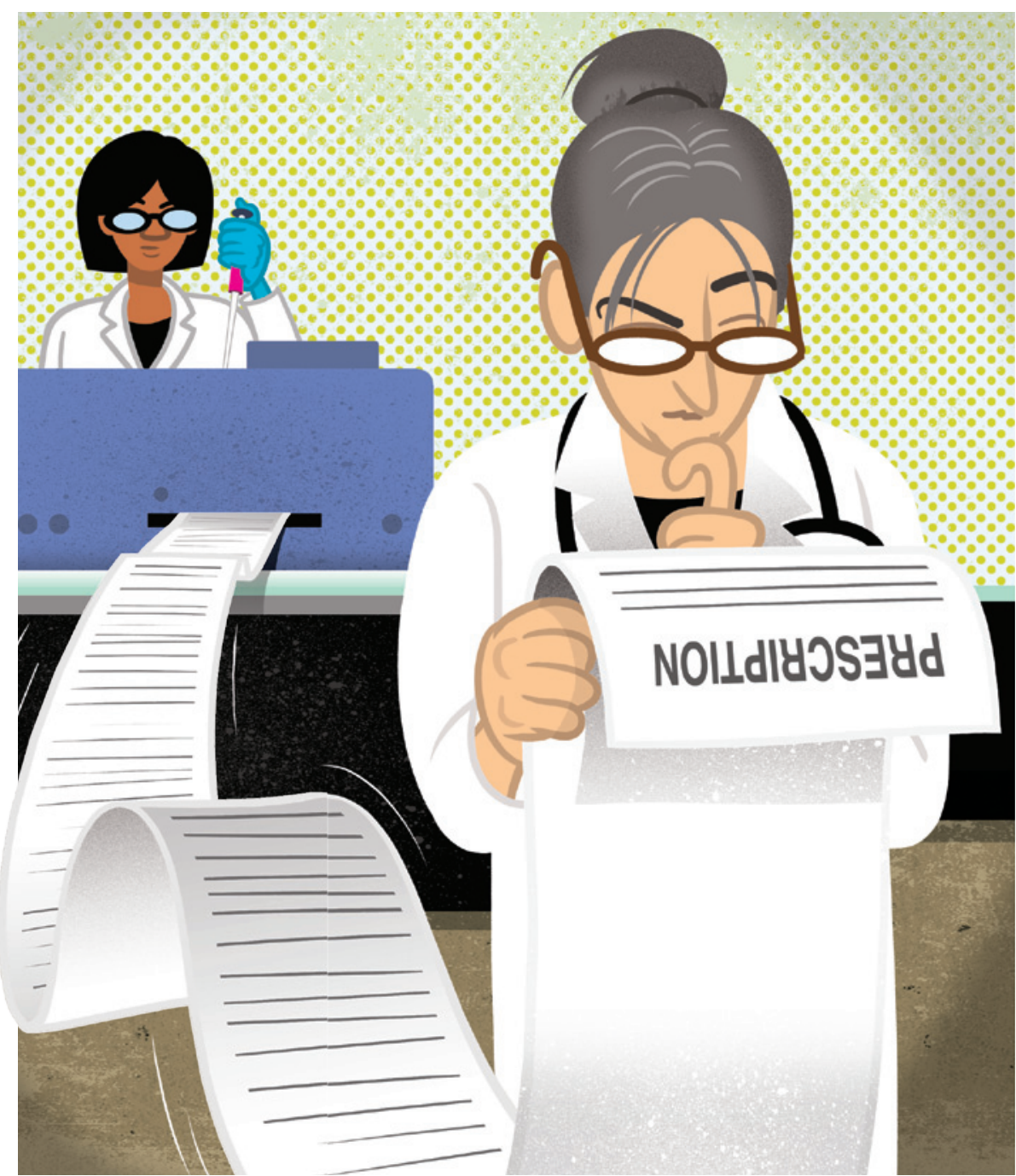

in diagnostic laboratories. It is performed intermittently over patient's lives to detect the emergence of viruses resistant to therapy. Sequencing for other viruses is also likely in the near future. With a raft of new drugs to treat hepatitis B and hepatitis C entering the market, detecting resistance will be required for hepatitis drug trials and clinical care.

\section{AUTOMATED ANALYSIS}

The major barrier to bringing microbial sequencing to the clinic is the lack of automated analysis tools. Once laboratory technicians have sequenced a pathogen, they need to convert the data into information that can be understood by non-specialists.

A probable scenario is that registered clinical users will access a web-based system. This is the model used for HIV interpretation. The HIVdb Program at Stanford University in California accepts user-submitted sequences of key genes and predicts levels of resistance to commonly used drugs. Analysis of other pathogens is likely to follow suit: submitted genetic data will be compared to a reference database of known variants and how they affect drug susceptibility. To be effective, reference databases will require considerable upkeep: new variants must be incorporated and tested for their effects on susceptibility.

The development of a single interpretation pipeline for all microbial sequence data will be impossible at first. For example, detecting resistance in bacteria that cause an acute infection is different from doing so in viruses that cause long-term infection. The type of analysis needed to predict resistance is also different from that required to investigate an outbreak. Specifically, instead of scanning for established resistance markers, newly sequenced genomes have to be compared against others to assess whether a related genome had been observed previously within a hospital, and if so when and where.

Academic and commercial competition is likely to lead to the emergence of a suite of excellent tools. However, data will be more powerful if they can be brought together. The collation of microbial sequences generated in a country could provide national surveillance on the emergence and spread of antimicrobial resistance. Because antimicrobial resistance is a global problem, further linkage will be needed to produce an international database, an unprecedented opportunity to detect resistance and new disease threats. It could also serve as an early-warning system for the emergence of strains not controlled by existing vaccines.

\section{FINDING FUNDING}

Although the potential benefits of an international network are clear, funding is less so. The translation of microbial sequencing into the clinic has largely been supported by short-term research funding to validate technology and timelines. Sustaining a longterm international programme will require a different funding model.

Launched in 2011, the Global Microbial Identifier is an initiative to create a genomic epidemiological database to identify microorganisms, which could be used to detect outbreaks, antimicrobial resistance and emerging pathogens. Experts from more than 30 countries have signed on, and the effort is attracting interest from funders, governments and academia.

An essential issue will be deciding who is permitted to access microbial genome data generated during clinical care. Sharing data with the research community and pharmaceutical companies would be useful for developing drugs and evaluating interventions, but will require safeguards to protect the identity of individuals. Analysis of microbial genomes could be used to try and reveal who gave which disease to whom, which is especially sensitive for sexually transmitted diseases. The use of viral gene-sequence data in the law court to prove that HIV transmission occurred directly between two individuals is unsound and unwelcome ${ }^{5}$. Failsafe systems that prevent data from being accessed by unauthorized individuals are obligatory.

The application of sequencing technology to microbial genomes will improve patient care and enhance public health. The feasibility and economics are clear. To reap these benefits, the logistics must be established. With automated analysis tools and laboratory procedures in place, sequencing can help patients, stall outbreaks and help to stave off the post-antibiotic era.

Sharon Peacock is professor of microbiology at the University of Cambridge, UK, and an honorary consultant microbiologist for Public Health England and the Cambridge University Hospitals NHS Foundation Trust. e-mail:sjp97@medschl.cam.ac.uk

1. Köser, C. U. et al. N. Engl. J. Med. 369, 290-292 (2013).

2. Reuter. S. et al. JAMA Intern. Med. 173, 13971404 (2013).

3. World Health Organization The Evolving Threat Of Antimicrobial Resistance: Options For Action (WHO, 2012).

4. Snitkin, E. S. et al. Sci. TransI. Med. 4, 148ra116 (2012).

5. Bernard, E. J. et al. HIV Med. 8, 382-387 (2007). 